\title{
Ist im Krieg gegen Terrorismus alles erlaubt?
}

\author{
Gerhard Beestermöller*
}

\begin{abstract}
The new law on air traffic security permits shooting down civil airplanes if the current state of affair indicates that the airplane is to be used as an instrument of a terrorist attack, and this attack could not be prevented by other means. One is also determined to kill the people in the airplane in such a case since they cannot be saved and in order to prevent more victims from dying as a result of a terrorist attack. The new law therefore stands in contrast to previous notions of basic rights that would not balance the life of one innocent person against the life of a larger number of equally innocent people. Even the argumentative figure of indirect killing does not help justify shooting down civil airplanes. A broad debate is needed on the limits of the legitimate use of violence for countering terrorism.
\end{abstract}

Keywords: Luftsicherheitsgesetz, Grundrechte, Prinzip der Doppelwirkung einer Handlung, Tötung Unschuldiger.

$\mathrm{S}$ tellen wir uns folgenden Fall vor: Einer Gruppe von Terroristen gelingt es, sich eines Flugzeuges zu bemächtigen. Das Flugzeug nähert sich Frankfurt am Main. Aus den abgehörten Gesprächen im Cockpit wird zweifelsfrei deutlich, dass die Terroristen das Flugzeug in den Messeturm lenken werden. Zur gleichen Zeit befindet sich eine Maschine der Luftwaffe in hinreichender Nähe zu dem entführten Luftfahrzeug und verfügt über die notwendige Bewaffnung, dieses abzuschießen. Würde die Passagiermaschine nicht unter Beschuss genommen, würden mit größter Wahrscheinlichkeit sowohl die Fluggäste als auch die vielen Menschen, die sich im Messeturm aufhalten, zu Tode kommen. Würde hingegen das Flugzeug abgeschossen, würden ausschließlich dessen Insassen - das sei hier einmal unterstellt - sterben. Was spricht dagegen, den Piloten der Luftwaffe einen entsprechenden Befehl $\mathrm{zu}$ erteilen? Warum soll es verboten sein, unschuldige Menschen zu retten und dabei anderen das Leben zu nehmen, wenn man unterstellen muss, dass diese schon so gut wie tot sind?

Nach Meinung des Bundestages jedenfalls gibt es keine zwingenden Gründe, ein derartiges Flugzeug nicht abzuschießen: »Die unmittelbare Einwirkung mit Waffengewalt ist nur zulässig, wenn nach den Umständen davon auszugehen ist, dass das Luftfahrzeug gegen das Leben von Menschen eingesetzt werden soll, und sie das einzige Mittel zur Abwehr dieser gegenwärtigen Gefahr ist." So die entscheidenden Worte des neuen »Gesetz(es) zur Neuregelung von Luftsicherheitsaufgaben «( 14 Absatz 3), das am 24. September 2004 vom Bundestag beschlossen wurde. Es soll dem Staat erlauben, unter bestimmten Umständen unschuldige Menschen zu töten, deren Leben nicht gerettet werden kann, um das Leben anderer unschuldiger Menschen zu retten.

Was ist gegen eine derartige Abwägung zu sagen? Stellt sie nicht einfach eine Forderung des gesunden Menschenverstandes dar? Folgt man der Argumentation des Hambur-

\footnotetext{
* Prof. Dr. Gerhard Beestermöller, stellvertretender Direktor am Institut für Theologie und Frieden (IThF), Barsbüttel.
}

ger Rechtsphilosophen Reinhard Merkel, ist das keineswegs der Fall. Für ihn läuft der Gesetzentwurf darauf hinaus, dem Staat zu erlauben, angesichts einer äußersten Bedrohung Menschen aus dem Bereich des Rechts auszuschließen, was die grundgesetzliche Ordnung der Bundesrepublik aufs Schwerste verletze (DIE ZEIT, 8. 7. 2004).

In der Tat stellt auch m.E. eine Legalisierung der Tötung Unschuldiger und darüber hinaus durch den Garanten des Rechts selbst unter allen denkbaren Bedingungen einen schweren Bruch unserer Rechtsordnung dar. Denn es ist das Wesen individueller Grundrechte, dass sie sich jeder Verrechnung sowohl mit den Rechten anderer Einzelner als auch sogar mit dem Bestand des Gemeinwesens entziehen.

Die Argumentation von Merkel lässt sich in diesem Punkt noch verstärken. Die Tötung Unschuldiger ist auch dann nicht rechtfertigungsfähig, wenn den todgeweihten Menschen, die zum Schutz anderer durch den Staat sozusagen vorab getötet werden sollen, nur noch einige Minuten zu leben bleiben. Wie viele der am 11. September $2001 \mathrm{zu}$ Tode Gekommenen haben vielleicht in den letzten Sekunden ihres Lebens ihren Lieben die entscheidenden, erlösenden Worte, $\mathrm{zu}$ denen sie sich jahrelang nicht haben durchringen können, über ihr Handy noch sagen können? Aber auch dann, wenn es in dem Flugzeug neben den Terroristen nur einen einzigen Menschen geben sollte, der seit Jahrzehnten im Koma und der darüber hinaus im Sterben liegt, lässt sich ein Abschuss dieser Maschine nicht legitimieren. Denn die Menschenwürde und das aus ihr erwachsene Lebensrecht haben ihren Grund darin, dass der Mensch eine zur Sittlichkeit fähige Person ist. Nur deshalb hat sein »Dasein an sich selbst einen absoluten Wert« (Kant). Würde man diesen Wert an irgendein Kriterium binden, das ein Mensch über seine schlichte Zugehörigkeit zur Spezies Mensch hinaus erfüllen kann oder nicht, und sei es ein Minimum an Lebenserwartung, würde man eine Setzung vornehmen. Der Wert des Menschen wäre dann nur noch eine relative Größe, nämlich abhängig von der Entscheidung dessen, der diese Kriterien festlegt. 
In diesem Punkt sehe ich keine Differenz zwischen dem Hamburger Rechtsphilosophen und mir. Hingegen scheint er aber der Ansicht zu sein, dass sich der Abschuss eines Passagierflugzeuges in Szenarien der beschriebenen Art letztlich doch rechtfertigen lasse. Das möchte ich entschieden bestreiten. Die direkte Tötung unschuldiger Menschen ist m.E. unter keinen Umständen erlaubt.

Ich bin mir sehr wohl darüber im Klaren, dass dies starke Worte sind. Ich bin mir auch durchaus der Tragik bewusst, die Situationen der beschriebenen Art bedeuten. Es bedarf keiner weitschweifender Erläuterungen, um die Gewissensnot derjenigen zu erkennen, die für unsere gesetzliche Ordnung Verantwortung zu tragen haben, und derjenigen, die im kritischen Augenblick über das Leben vieler, vieler unschuldiger Menschen entscheiden müssen - so oder so. Das alles kann aber nicht davon abhalten, sondern muss uns gerade dazu zwingen, die Dinge zwar mit glühendem Herzen, aber eben mit kühlem Verstand konsequent zu bedenken.

Müssen wir also tatenlos zusehen, wie das Flugzeug in den Messeturm stürzt? Alles in uns sträubt sich gegen diese Schlussfolgerung. Merkel jedenfalls sucht nach einer Möglichkeit, einen Abschuss am Ende doch legitimieren zu können; dann nämlich, wenn von derartigen terroristischen Aktionen die staatliche Rechtsmacht in ihrem Fortbestand bedroht wird. Die Erhaltung der friedens- und rechtsichernden Macht des Staates ist eine Bedingung sine qua non seiner Legitimität. Wenn also der Staat als äußerstes Mittel seiner Selbsterhaltung Unschuldige tötet, dann ist er, so muss man die Argumentation wohl interpretieren, gerechtfertigt. Es ehrt den Hamburger Rechtsphilosophen, dass er keinen Zweifel daran aufkommen lässt, dass eine derartige Auffassung mit unserem Grundgesetz und den es repräsentierenden Grundnormen bricht. Denn es würde bestimmte Menschen aus dem Kreis der Grundrechtsträger ausschließen.

Mir scheint, dass man diese Argumentation an diesem Punkt noch verschärfen muss. Wer unschuldige Menschen unter bestimmten Bedingungen aus dem Kreis des Rechts ausschließt, schließt alle aus, die diese Bedingungen erfüllen, und, da alle zu diesem Kreis gehören könnten: jeden! Wer dies legitimieren will, und zugleich zugibt, dass es sich im Sinnzusammenhang unserer Rechtsordnung nicht legitimieren lässt, hat nur zwei Möglichkeiten. Eine erste Alternative besteht darin zuzugeben, dass die direkte Tötung Unschuldiger einen direkten Bruch mit den grundlegenden Normüberzeugungen darstellt, für die unsere Ordnung steht. Die zweite Alternative besteht darin zu behaupten, es gäbe sozusagen oberhalb des Normensystems, auf dem unser Staat ruhe, eine übergreifende Legitimationsebene. Unser Normensystem stelle im Blick auf diese Ebene sozusagen eine erste Anwendung dar, die für die allermeisten Fälle gelte. Daneben gäbe es aber noch Fälle wie der unseres Flugzeugs. Eine Anwendung der obersten Normebene auf diese Fälle würde eben zu Handlungsanweisungen jenseits der Möglichkeiten unserer Ordnung führen. Auch diese Alternative stellt einen Bruch mit unseren Grundnormen dar, die sich sozusagen als Erst- bzw. Letztgrößen verstehen.
Vielleicht ein wenig zu spitz formuliert, läuft die Argumentation Merkels darauf hinaus, dass im Krieg gegen den Terrorismus alles erlaubt ist, was zur Sicherung des Bestandes des Staates notwendig ist. Der Unterschied zur klassischen Kriegsethik besteht im wesentlichen darin, dass es dort in der Regel um die Frage geht, in welchem Umfang man die Zivilbevölkerung des Staates, gegen den man Waffengewalt richtet, in Mitleidenschaft ziehen darf. Hier hingegen läuft es auf die Frage hinaus, inwiefern man legitimiert ist, unschuldigen Menschen der eigenen Bevölkerung in der gewaltsamen Bekämpfung des Terrorismus das Leben zu nehmen. Merkel, so scheint es mir, bricht mit der Tradition des sogenannten ,ius in bello', in dem es darum geht, dem Staat in der Wahl seiner Mittel auch in äußerster Selbstverteidigung Grenzen zu ziehen.

Hier drängt sich sofort eine Frage auf: Wie soll sich ein Staat mit Waffengewalt verteidigen können, ohne auch nur einen einzigen unschuldigen Menschen zu töten? Läuft diese Forderung nicht darauf hinaus, eine kampflose Kapitulation $\mathrm{zu}$ fordern? Kann man eine derartige Forderung allen ethischen Ernstes erheben?

Nun, die klassische Kriegsethik fordert keineswegs, jedwede Gewaltanwendung schlechthin $\mathrm{zu}$ unterlassen, die nach menschlichem Ermessen Unschuldigen das Leben nimmt. Sie sucht vielmehr nach einem schmalen Grat zwischen Scylla und Charybdis, nämlich auf der einen Seite Menschen schutzlos ungerechter Gewalt zu überlassen und auf der anderen Seite auch nur einen Menschen zu instrumentalisieren.

Um hier Grund unter die Füße zu bekommen, unterscheidet das klassische ius in bello zwischen der direkten und der indirekten Tötung von Unschuldigen. Demzufolge ist es ethisch ein himmelweiter Unterschied, ob ein unschuldiger Mensch in Folge einer kriegerischen Handlung zu Tode kommt, die sich direkt gegen ihn richtet, oder ob sein Tod als deren Nebenfolge eintritt.

Stellen wir uns vor, wir könnten einen Terroristen nur dadurch von einem schweren Anschlag abhalten, in dem wir ihn hier und jetzt in die Luft jagen. Wir nehmen uns vor, nicht mehr Sprengstoff zu verwenden als unbedingt notwendig. Zugleich wissen wir, dass es dennoch eine unvermeidbare Splitterwirkung geben wird, die ein in der Nähe spielendes Kind töten wird. Folgt man dem Prinzip der Doppelwirkung einer Handlung, wäre für die sittliche Bewertung dieser Tat die Tötung des Terroristen und damit die Verhinderung weiterer Terroranschläge und nicht der Tod des Kindes entscheidend. Wer so handelt, verletzt nicht auf illegitime Weise das Lebensrecht des unschuldigen Kindes.

Bei Handlungen mit Todesfolge von Nebenwirkungen zu sprechen, hat in der letzten Zeit eine schlechte Presse. Vor wenigen Jahren wurde gar das Wort »Kollateralschaden« zum Unwort des Jahres erkoren. Es soll nicht bestritten werden, dass es die Möglichkeit einräumt, beispielsweise die Tötung von unschuldigen Menschen im Krieg auf missbräuchliche Weise zu rechtfertigen. Natürlich kann man eine Kriegshandlung, die eine ganze Stadt in Schutt und Asche legt, nicht dadurch legitimieren, dass sie aus der In- 
tention geführt wurde, ein bestimmtes militärisches Ziel innerhalb des Stadtgebietes $\mathrm{zu}$ treffen. Im rechten Gebrauch des Begriffs geht es vielmehr gerade darum, das Argument abzuwehren, durch Verweis auf die gute Intention des Handelnden jedwede Schadensdimension legitimieren zu können.

Die Tötung von Unschuldigen ist aber nicht schon dann legitim, wenn sie sich als eine Nebenwirkung einer rechtfertigbaren Hauptwirkung darstellen lässt. Vielmehr fordert das Prinzip der Doppelwirkung als zweite Bedingung, dass beide Wirkungen zugleich aus der Handlung hervorgehen. Die dritte Bedingung ist, dass sich der Haupteffekt der Handlung nicht auf andere, sittlich weniger problematische Weise erreichen lässt. Die vierte Bedingung besteht darin, dass die positiven Wirkungen im Verhältnis zu den negativen stehen. Erst die fünfte Bedingung hebt auf die Intention des Handelnden ab. Er handelt nur dann schuldfrei, wenn er ausschließlich durch die positiven Wirkungen motiviert ist und die negativen nur bedauernd in Kauf nimmt.

Natürlich kann man das Prinzip der Doppelwirkung in Frage stellen. Vielleicht muss man das auch. Wird die Unverrechenbarkeit eines jeden menschlichen Lebens hier wirklich gewahrt? Soviel lässt sich aber sagen: Wenn sich der Abschuss einer zivilen Maschine nicht einmal mittels dieses Prinzips rechtfertigen lässt, dann zeigt sich überhaupt kein Ansatz für eine mögliche Rechtfertigung. Der Abschuss muss dann $\mathrm{m}$. E. als verwerflich gelten und unterbleiben, wenn man denn daran festhalten will, dass auch im Krieg nicht alles erlaubt ist, dass auch der Krieg gegen Terrorismus keine grundwertefreie Zone schafft.

Ließe sich nun der Abschuss einer Passagiermaschine mittels des Prinzips der Doppelwirkung rechtfertigen? Ich denke nicht, obwohl der Tod der Passagiere und der Terroristen in keinem kausalen Verhältnis miteinander verbunden ist, die Rettung der einen und der Tod der anderen zugleich aus der Handlung hervorgehen, sehr viele Menschen gerettet werden können und die Anweisenden und Ausführenden den Tod der Unschuldigen nur bedauernd in Kauf nehmen, was man ohne weiteres unterstellen kann! Denn wer ein Flugzeug abschießt, tötet Terroristen und Passagiere unterschiedslos in einem. Es mag in vielen Fällen schwierig sein zu bestimmen, ob man noch zwischen Haupt- und Nebenwirkungen unterscheiden kann oder nur von einer einzigen Wirkung sprechen muss. Im Fall des Flugzeugabschusses scheint mir die Sache genauso eindeutig wie im Fall eines Hauses, in dessen Keller sich Soldaten befinden. Dieses Haus samt seinen Bewohnern in die Luft zu sprengen kann nicht mittels der Kategorie der Nebenfolge einer legitimen Kriegshandlung gerechtfertigt werden.

Daher ist der Abschuss eines zivilen Flugzeuges in Szenarien der geschilderten Art nicht zu legitimieren, ohne die rechtsmoralische Identität unseres Gemeinwesens aufzugeben und ohne mit jenen Traditionen $\mathrm{zu}$ brechen, in denen sie wurzelt. Alles in uns sträubt sich gegen diese Konsequenz - aber gegen den Abschuss von Passagierflugzeugen doch wohl nicht weniger!

Was wir heute dringend brauchen, ist eine breit geführte Debatte über die Grenzen legitimer Gewalt. Es sei nur an die Beiträge erinnert, die die Absolutheit des Folterverbotes in Zweifel ziehen. Im Grunde brauchen wir eine neue Grundwertedebatte. Es ist zu vermuten, dass die CDU/CSU vor das Bundesverfassungsgericht ziehen wird. Vielleicht findet das Thema auf diese Weise die notwendige Aufmerksamkeit, die es bisher erstaunlicherweise nicht gefunden hat. Wir sollten uns Zeit lassen, zuviel steht auf dem Spiel! 